\title{
Malformación arteriovenosa gigante de cuero cabelludo: caso clínico
}

\author{
M. García-Conde; L. Martín-Viota; P. Febles-García; S. Cortés-Franco; A.M. Millán-Corada; M. Spreafico-Guerrero; \\ E. Prada y V. García-Marín
}

Servicio de Neurocirugía. Hospital Universitario de Canarias. La Laguna. Santa Cruz de Tenerife.

Resumen

Las malformaciones arteriovenosas (MAV) en el cuero cabelludo son lesiones vasculares muy infrecuentes. Sus síntomas son variados, desde molestas y antiestéticas tumoraciones de la piel, hasta hemorragias que pueden ser devastadoras. Su origen puede ser congénito o traumático. El diagnóstico de las mismas es clínico, mediante la inspección del enfermo, y la angiografía de las carótidas interna y externa confirma el diagnóstico. Las posibilidades terapéuticas son variadas, siendo la extirpación quirúrgica el tratamiento de elección, aunque cada vez se utiliza más el tratamiento endovascular como tratamiento único o prequirúrgico, para disminuir las pérdidas hemáticas.

Presentamos el caso de una paciente de 50 años de edad que presentaba una gran tumoración en cuero cabelludo, con dilataciones vasculares muy marcadas y sin otra sintomatología asociada. Describió un antecedente traumático hacía 12 años. La angiografía mostró una MAV abigarrada, con aporte vascular de la carótida externa y también de la interna, a cargo de ramas meníngeas transoseas provenientes de arterias etmoidales y dependientes de ambas arterias oftálmicas. El tratamiento endovascular de los aportes intracraneales no fue posible, debido al alto riesgo de amaurosis uni o bilateral, por lo que la lesión se resecó quirúrgicamente, sin presentarse complicaciones.

El tratamiento de las MAV de cuero cabelludo ofrece diversas posibilidades pero se hace imprescindible la individualización de cada caso a la hora de decidir el abordaje correcto para evitar las posibles complicaciones.

PALABRAS CLAVE: Malformación arteriovenosa. Aneurisma cirsoideo. Cuero cabelludo. Tratamiento endovascular

Giant arteriovenous malformation of the scalp. Case report

Recibido; 23-08-05. Aceptado: 19-10-05
Summary

Arteriovenous malformations (AVM) in the scalp are infrequent vascular lesions. Its clinical presentation varies from annoying and unaesthetic mass of the skin to devastating hemorrhages. Its origin can be congenital or traumatic. The diagnosis of AVM is based on physical examination and confirmed by internal and external carotid angiography. Nowadays the gold standard treatment is the surgical intervention although the endovascular approach is gaining field in order to reduce blood losses as presurgical or like lonely treatment.

A 50 year old woman was admitted with a huge mass in scalp, with subcutaneous enlarged vessels and no other symptoms. A head traumatic antecedent had occurred 12 years before. The angiography evidenced a mottled AVM with blood supplies from the external and internal carotid arteries, with meningeal transosseous branches from both ophthalmic arteries. Endovascular treatment could not be performed due to high risk of uni- or bilateral amaurosis. Thus, a conventional surgical treatment was done without complications. The treatment of AVM of scalp offers various possibilities but the individualization of each case becomes essential to decide the correct management in order to avoid complications.

KEY WORDS: Arteriovenous malformation. Cirsoid aneurysm. Scalp. Endovascular treatment.

\section{Introducción}

Las malformaciones arteriovenosas del cuero cabelludo son unas lesiones vasculares muy infrecuentes con manifestaciones clínicas muy variadas. Producen una deformidad pulsátil en el cuero cabelludo que crece progresivamente, y además un aumento de tamaño de los diferentes vasos arteriales y venosos circundantes. Otros síntomas pueden ser cefalea ${ }^{5,20}$, necrosis de la piel suprayacente ${ }^{20}$, hemorragia $^{11,12,20}$, que puede ser incluso fatal ${ }^{6}$, alopecia ${ }^{15}$, o incluso crisis convulsivas y retardo psicomotor ${ }^{16} \mathrm{o}$ fallo cardíaco 


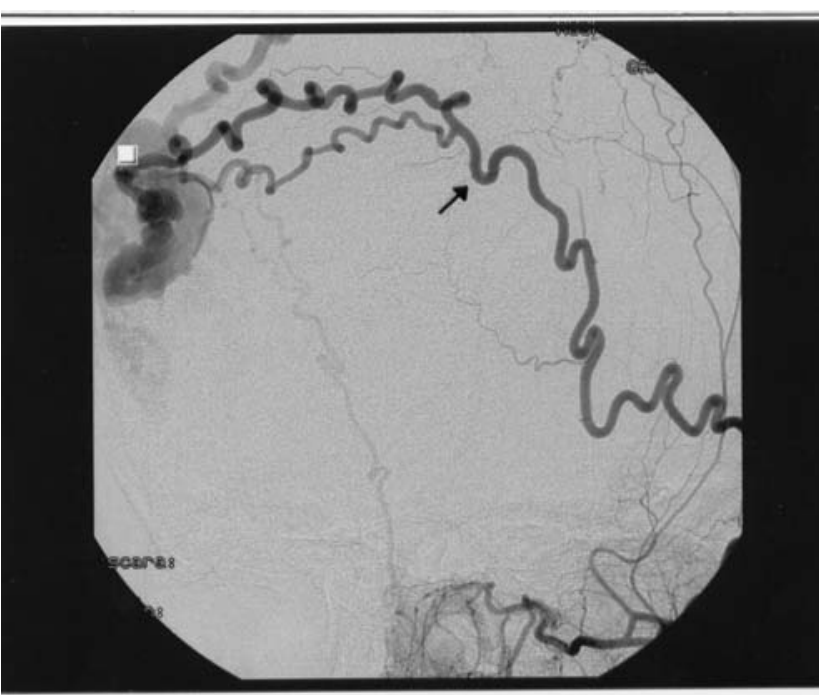

Figura 1. Proyección anteroposterior. Aporte a cargo de la arteria temporal superfical izquierda.

por alto flujo ${ }^{23}$. El manejo de estas lesiones requieren confirmación diagnóstica, como mínimo, mediante angiografía cerebral y el tratamiento que se propone es agresivo, puesto que su tendencia natural es el crecimiento progresivo. El tratamiento que clásicamente se ha utilizado ha sido la resección quirúrgica. Otras alternativas son el tratamiento endovascular con embolización, la radiocirugía, la electro-trombosis, la inyección con sustancias esclerosantes $\mathrm{y}$, como variante al tratamiento quirúrgico, la resección completa de partes blandas con la MAV seguida de trasposición de piel con injertos autólogos. El tratamiento de elección continúa siendo la resección quirúrgica, si bien el tratamiento endovascular ayuda a disminuir la hemorragia perioperatoria y en algunos casos, sobre todo en MAV pequeñas se ha demostrado curativa.

Presentamos el manejo de una malformación arteriovenosa de gran tamaño y múltiples aportes tratada quirúrgicamente en nuestro Servicio, hacemos una revisión bibliográfica y comentamos los aspectos clínicoradiológicos de esta rara entidad.

\section{Caso clínico}

Presentamos el caso clínico de una mujer de 50 años de edad, de profesión equilibrista funambulista, sin antecedentes personales de interés, que desarrolló progresivamente, en el curso de unos 10 años, una malformación del cuero cabelludo consistente en una masa irregular frontal izquierda, de aproximadamente $12 \times 11 \mathrm{~cm}$ en sus ejes principales, pulsátil, con importante desarrollo de la trama vascular de la calota craneal, a cargo de las arterias temporales superficiales y sus ramas venosas de retorno. En la anamnesis, la paciente recuerda un antecedente

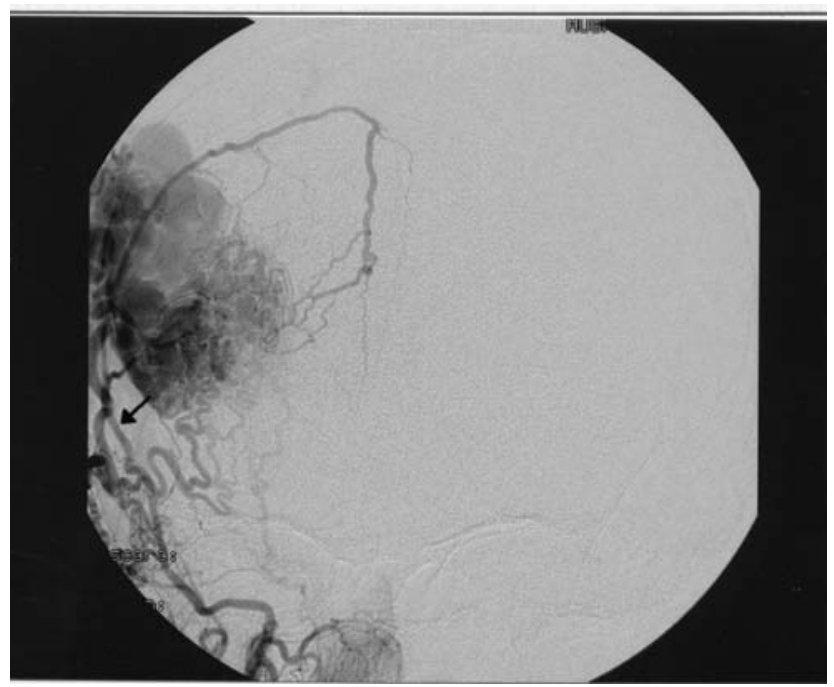

Figura 2. Proyección anteroposterior. Aporte a cargo de la arteria temporal superficial derecha.

traumático hacía 12 años, con contusión frontal, tras lo cual sufrió un importante hematoma subgaleal bifrontal, que se resolvió de manera conservadora en el curso de aproximadamente dos meses. Aparte de este incidente, no refiere otra sintomatología y la exploración física y neurológica es normal. Ante la sospecha de malformación arteriovenosa traumática se solicitan pruebas de imagen. En la RM cerebral no se evidenciaron alteraciones significativas y en la angiografía cerebral de los territorios de la arteria carótida interna y externa se demostró un nido vascular epicraneal frontal izquierdo, con aportes vasculares extracraneales a cargo de ambas arterias temporales superficiales (Figuras 1 y 2), y aportes provenientes de la circulación intracraneal a cargo de ramas meníngeas transoseas provenientes de las arterias etmoidales y dependientes a su vez de las dos arterias oftálmicas, izquierda (Figura 3) y derecha (Figura 4). No se llevó a cabo la embolización ni de las ramas provenientes de los aportes intracraneales debido al alto riesgo de amaurosis de uno o ambos ojos, ni de las extracraneales, por considerarse posible la resección de la MAV mediante la intervención quirúrgica exclusivamente. Se realizó un incisión bicoronal, por detrás del nido, accediendo bilateralmente a las ramas nutricias de la MAV, para primero ligarlas y seccionarlas y así disminuir el aporte sanguíneo a la lesión y hacer posible la resección de la misma con un sangrado controlado. El nido se encontraba por fuera del pericráneo y bajo el tejido celular subcutáneo, y mostraba, irrigación a través de la calota, desde las ramas intracraneales que evidenciaba la angiografía previa. Se utilizó coagulación por radiofrecuencia, tanto mono como bipolar y cera de hueso para controlar la hemorragia. Se realizó la resección de todos los vasos sanguíneos subcutáneos, y sólo en algunas zonas aisladas y puntuales 

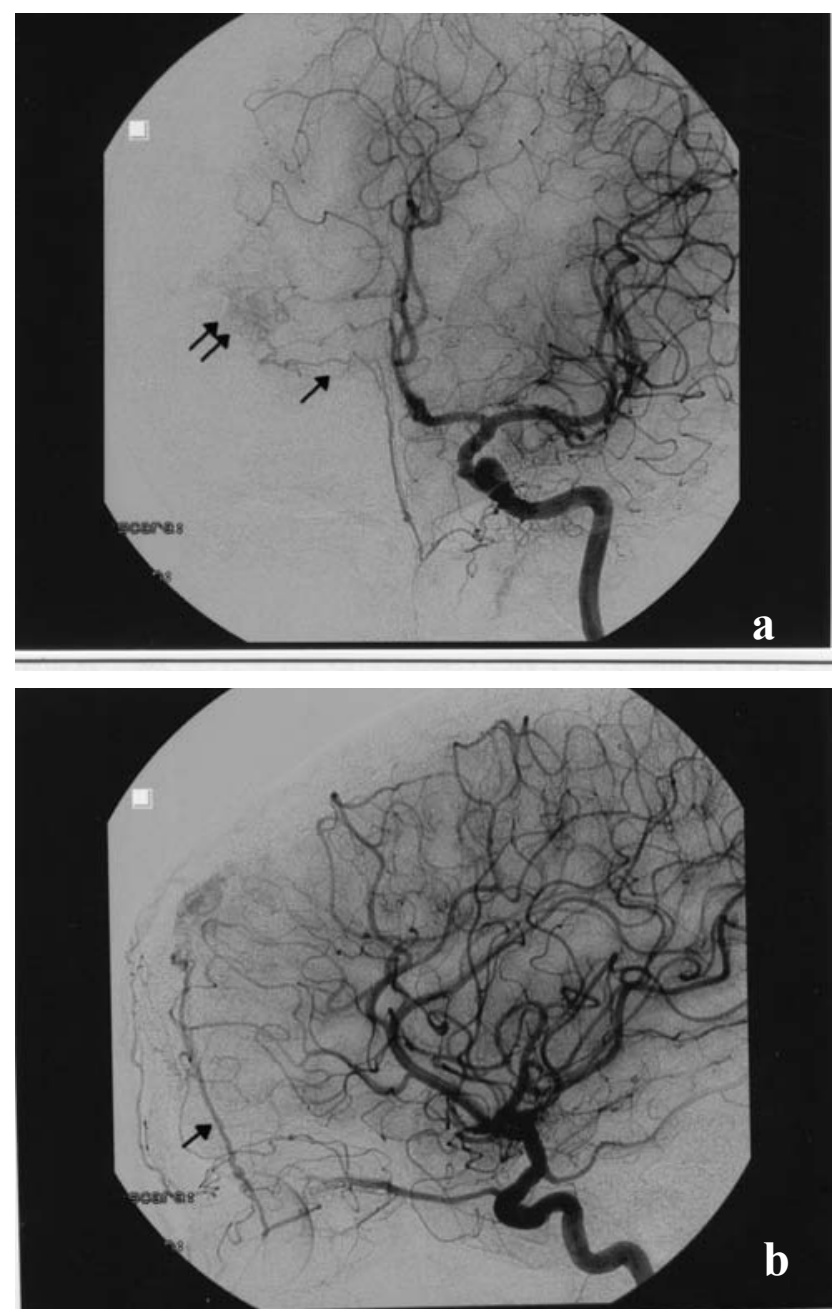

Figura 3. Ramas meningeas transóseas, drenando en el nido (doble flecha), provenientes de las arterias etmoidales (flecha) y dependientes de la arteria oftálmica izquierda en proyecciónes anteroposterior (a) y lateral (b).

llegaron a evidenciarse folículos pilosos. Durante la cirugía fue preciso transfundir con dos concentrados de hematíes, utilizándose, además, un recuperador sanguíneo (ORTHOPAT HAEMONETICS) para un mejor control de la pérdida de sangre. El cuero cabelludo se suturó a la manera habitual por planos y la piel con agrafes. Durante el postoperatorio no se produjeron complicaciones neurológicas y la herida cicatrizó adecuadamente, sin desarrollar zonas de necrosis ni alopecia. El resultado cosmético fue excelente. Después de un año no ha mostrado recidiva.

\section{Discusión}

Las malformaciones arteriovenosas del cuero cabelludo fueron descritas por primera vez por Hunter, en 1757. Posteriormente, Brecht, las denominó aneurismas cirsoideos.

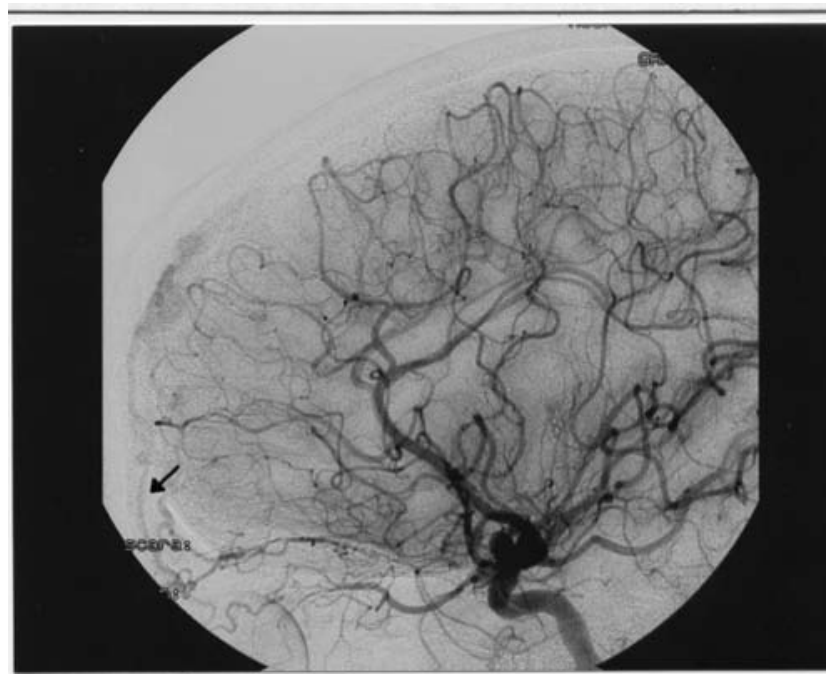

Figura 4. Proyección lateral. Ramas meníngeas transóseas provenientes de las arterias etmoidales, dependientes de la arteria oftálmica derecha.

A lo largo de los años han recibido otros muchos nombres como aneurismas arteriovenosos, fístulas arteriovenosas, aneurismas racemosos y angiomas plexiformes.

Estas lesiones constan de unas ramas arteriales nutricias $\mathrm{y}$ unas venas de drenaje, sin existir entre ellas un lecho capilar, sino un nido donde se encuentra la fístula arteriovenosa, y a veces otros componentes, también descritos, como hemangiomas o fístulas plexiformes ${ }^{20}$. Las ramas arteriales que nutren la malformación proceden en la mayoría de los casos de ramas de la arteria carótida externa y en algunas situaciones, como en nuestro caso, de arterias procedentes de la carótida interna. El drenaje venoso se produce normalmente en el sistema venoso del cuero cabelludo pero también se ha descrito en senos venosos intracraneales ${ }^{12,21}$. $\mathrm{Al}$ igual que lo descrito por otros autores, nosotros también encontramos un componente pericraneal ${ }^{3}$.

Tal como se demostró en un estudio experimental en $\operatorname{ratas}^{27}$ el crecimiento de la MAV se ve promovida por la producción de factores angiogénicos debido a que la hipertensión venosa reduce la perfusión del tejido y se produce isquemia tisular. Una actividad aberrante angiogénica promueve el shunt y la formación de nuevas fístulas arteriovenosas. Los cambios hemodinámicos inducen el aumento progresivo de las varices venosas, con el consiguiente aumento de la masa ${ }^{5}$. Es tan grande el estrés hemodinámico que se ha descrito también, asociado a estas malformaciones, la aparición de aneurismas en una de las ramas nutricias ${ }^{15}$. La malformación vascular puede no ser sólo extracraneal y en ocasiones tener asociada una malformación arteriovenosa intracerebral ${ }^{9,12,30}$, e incluso aparecer en el contexto de una enfermedad familiar con malformaciones arteriovenosas múltiples ${ }^{1,24}$. 
Aparecen con mayor frecuencia a nivel temporal, frontal y occipital $^{5}$ y sus ramas nutricias son, en orden decreciente de frecuencia, la arteria temporal superficial, occipital y auricular posterior ${ }^{19}$.

En cuanto a su origen pueden ser congénitas y traumáticas, siendo las primeras las más frecuentes. Sobre todo, cuando aparecen en niños, se debe establecer un diagnóstico diferencial entre sinus pericranii, encefalocele, hamartomas vasculares, hemangiomas, etc ${ }^{5}$. Dentro de las traumáticas, el desarrollo es normalmente muy lento, observándose un crecimiento relevante en un periodo de 6 a 12 años $^{5}$. Se han descrito malformaciones arteriovenosas también secundarias a craneotomía ${ }^{17}$, trasplante de pelo ${ }^{2,12,14,28}$, artroscopia de la unión temporomandibular ${ }^{5}$, etc.

En el diagnóstico se hace indispensable la angiografía de las ramas de la carótida externa e interna. La MAV se sospecha angiográficamente por los vasos prominentes y el relleno vascular temprano ${ }^{20}$. La angiografía cerebral digital es el estudio complementario de elección en esta patología y se hace indispensable para el estudio de estas lesiones y para descartar patología intracerebral asociada. Los estudios de RMN y AngioRM también pueden ser orientativos y ayudar a documentar de forma completa cada caso así como a planificar el tratamiento. Shenoy SN et al., dividen las malformaciones arteriovenosas en dos grupos, primario, si las arterias y el drenaje venoso pertenecen en su totalidad al cuero cabelludo, y secundarias, cuando estos vasos son el flujo exterior de malformaciones vasculares intracerebrales. Refieren el caso de un paciente de 25 años de edad clasificado erróneamente dentro del primer grupo que dos días después de la cirugía desarrolla un edema cerebral masivo y causa exitus ${ }^{22}$.

El manejo de las MAV de cuero cabelludo es difícil, y aunque a veces se decide actitud conservadora, dada la tendencia que presentan al crecimiento y al posible aumento de los síntomas ${ }^{15}$, parece más oportuno un tratamiento precoz. El tratamiento de elección es la resección quirúrgica extensa de toda la malformación y todas sus ramas nutricias $^{20}$, dada las posibilidad de recurrencia si no se elimina el aporte sanguíneo ${ }^{22}$. Se han mostrado recurrencias por insuficiente devascularización por contribución a cargo de la arteria oftálmica ${ }^{5}$. Dentro de las posibles complicaciones de la resección quirúrgica, se han descrito la necrosis del cuero cabelludo ${ }^{5,19}$ y sepsis por infección de la herida quirúrgica ${ }^{5}$. Diversos autores recomiendan un tratamiento endovascular asociado a la cirugía para disminuir el aporte sanguíneo de aquellas lesiones grandes, de manera que se facilite la intervención quirúrgica ${ }^{10,25}$, e incluso en las lesiones pequeñas, han demostrado efectividad del tratamiento endovascular aislado ${ }^{7,20}$. Como posible complicación de la embolización con coils está la necrosis de cuero cabelludo ${ }^{7}$. En nuestro caso se optó como tratamiento definitivo únicamente el abordaje quirúrgico, puesto que la embolización completa no era posible, debido al riesgo de amaurosis si se intentaba el tratamiento endovascular de las ramas intracraneales y dado que se consideraban abordables las ramas principales de aporte, a cargo de las arterias temporales superficiales izquierda (Figura 1) y derecha (Figura 2).

Otras posibilidades terapéuticas incluyen la embolización con sustancias esclerosantes, como el N-butyl-2cyanocrylato, considerado por algunos autores como el mejor material embolígeno ${ }^{14,20}$, sodio tetradecyl sulfato ${ }^{8}$ y alcohol etílico ${ }^{18}$. En situaciones en las que es imposible lograr una integridad del cuero cabelludo se han mostrado también efectivas las plastias con piel autóloga, por ejemplo del antebrazo. Mediante este injerto altamente vascularizado, que favorece el cambio histológico de las malformaciones arteriovenosas, se pretende bloquear el círculo vicioso de isquemia y reperfusión y favorecer así la curación ${ }^{4,26}$. También los expansores de tejido ayudan a la cicatrización de zonas donde el colgajo resulta insuficiente para cubrir la zona expuesta ${ }^{13,20}$.

\section{Conclusiones}

Las malformaciones arteriovenosas del cuero cabelludo deben ser consideradas como diagnóstico diferencial ante la aparición de una masa extracraneal. Al diagnóstico mediante la angiografía cerebral, puede añadirse la RM cerebral con secuencias angiográficas. Con ambas pruebas complementarias debe descartarse patología intracerebral coexistente, que añadiría mucha morbimortalidad al ya arriesgado tratamiento de esta patología. Ante el crecimiento progresivo y la posibilidad de aparición de sintomatología neurológica asociada, se recomienda un tratamiento precoz, combinando, de forma individualizada para cada caso, las técnicas endo-vasculares y quirúrgicas.

\section{Bibliografía}

1. Amin-Hanjani, S., Robertson, R., Arginteanu, M.S., Scott, R.M.: Familiar intracranial arteriovenous malformations: Case report and review of the literature. Pediatr Neurosurg 1998; 29: 208-213.

2. Davis, A.J., Nelson, P.K.: Arteriovenous fistula of the scalp secondary to punch autograft hair transplantation. Angioarchitecture, histopathology, and endovascular and surgical therapy. Plast Reconstr Surg 1997;100: 242-249.

3. Domingo, Z., Fisher-Jeffes, N.D., deVilliers, J.C.: Surgical management of arteriovenous malformations of the scalp. In Schmidek HN, editor. Operative neurosurgical techniques: Indications, methods and results. $4^{\text {th }}$ Ed. Philadelphia: Saunders Company; 2000. p. 1331-1338.

4. Dompmartin, A., Labbe, D., Barrellier, M.T., Theron, J.: Use of a regulating flap in the treatment of a large arteriovenous malformation of the scalp. Br J Plast Surg 1998; 51: 


\section{1-563.}

5. Fisher-Jeffes, N.D., Domingo, Z., Madden, M., de Villiers, J.C.: Arteriovenous malformations of the scalp. Neurosurgery $1995 ; 36$ : 656-660.

6. Gauger. G-E-, Herrmann. P.W.: Cranial arteriovenous malformation: suicide by exsanguination. J Forensic Sci 1988; 33: 283-286.

7. Heilman, C.B., Kwan, E.S., Klucznik, R.P., Cohen, A.R.: Elimination of a cirsoid aneurysm of the scalp by direct percutaneous embolization with thrombogenic coils. Case report. J Neurosurg 1990; 73: 296-300.

8. Hendrix, L.E., Meyer, G.A., Erickson, S.J.: Cirsoid aneurysm treatment by percutaneous injection of sodium tetradecyl sulfate. Surg Neurol 1996; 46 : 557-560.

9. Ishiguro, S., Kimura, A., Munemoto, S., Kitabayashi, M., Shoin, K., Futami, K.: Traumatic arteriovenous fistula with feeders of the scalp, duramater and pia mater: case report. No Shinkei Geka 1987;15: 677-681.

10. Komiyama, M., Nishikawa, M., Kitano, S., Sakamoto, H., Imai, K., Tsujiguchi, K., Mizuno, T.: Non-traumatic arteriovenous fistulas of the scalp treated by a combination of embolization and surgical removal. Neurol Med Chir (Tokyo)1996; 36: 162-165.

11. Kuroki, K., Taguchi, H., Sumida, M., Eguchi, K., Saitoh, Y.: A case of hemorrhagic non traumatic arteriovenous fistula of the scalp. No Shinkei Geka 1999; 27: 851-853.

12. Lanzino, G., Passacantilli, E., Lemole, G.M. Jr., McDougall, C., Spetzler, R.F.: Scalp arteriovenous malformation draining into the superior sagittal sinus associated with an intracranial arteriovenous malformation: just a coincidence? Case report. Neurosurgery 2003; 52: 440-443.

13. Marotta, T.R., Berenstein, A., Zide, B.: The combined role of embolization and tissue expanders in the management of arteriovenous malformations of the scalp. AJNR Am J Neuroradiol 1994; 150:1240-1246.

14. Mathis, J.M., DeNardo, A.J., Jensen, M.E., Lin, K.Y., Dion, JE.: Arteriovenous fistula of the scalp after hair transplantation treated by endovascular embolization. Ann Plast Surg 1994; 33: 633-637.

15. Matsushige, T., Kiya, K., Satoh, H., Mizoue, T., Kagawa, K., Araki, H.: Arteriovenous malformation of the scalp: case report and review of the literature. Surg Neurol 2004; 62: 253-259.

16. Mohanty, S., Rao, C.J.: A large cirsoid aneurysm of the scalp associated with epilepsy. J Neurol Neurosurg Psychiatry 1976; 39: 835-836.

17. Morioka, T., Nishio, S., Hikita, T.: Traumatic arteriovenous fistulae of the scalp at the area of previous craniotomy. Surg Neurol tie 1988; 30: 406-407.

18. Mourao, G.S., Hodes, J.E., Gobin, Y.P., Casasco, A., Aymard, A., Merland, J.J.: Curative treatment of scalp arteriovenous fistulas by direct puncture and embolization with absolute alcohol. Report of three cases. J Neurosurg 1991; 75:
634-637.

19. Muthukumar, N., Rajagopal, V., Manoharan, A.V., Durairaj, N.: Surgical management of cirsoid aneurysms. Acta Neurochir (Wien) 2002; 144: 349-356.

20. Nagasaka Susumu, M.D., Fukushima Takeo, M.D., Goto Katsuya, M.D., Ohjimi Hiroyuki, M.D., Iwabuchi Satoshi, M.D., Maehara Fumiaki, M.D.: Treatment of Scalp Arteriovenous Malformation. Neurosurg 1996; 38: 671-677.

21. Nishiura, T., Nishida, A., Handa, A., Gotoh, M., Tsuno, K., Ishimitsu, H.: Congenital arteriovenous malformation of the scalp with a drainage to the transverse sinus: a case report. No Shinkei Geka 1999; 27: 895-901.

22. Shenoy, S.N., Raja, A.: Scalp arteriovenous malformations. Neurol India 2004; 52: 478-481.

23. Sugrue, M., McCollum, P., O'Driscoll, K., Feeley, M., Shanik, D.G., Moore, D.J.: Congenital arteriovenous malformation of the scalp with high output cardiac failure: A case report. Ann Vasc Surg 1989; 3: 387-388.

24. Tamaki, N., Fujitak Yamashita, H.: Multiple arteriovenous malformations involving the scalp, dura, retina, cerebrum and posterior fosa: Case report. Neurosurg 1971; 31: 95-98.

25. Tanaka, T., Hasegawa, Y., Kanki, T., Hayashi, J., Ui, K., Usami, S., Abe, T.: Combination of intravascular surgery and surgical operation for occipital subcutaneous arteriovenous fistula in a patient with neurofibromatosis type I. No Shinkei Geka 2002; 30: 309-313.

26. Tark, K.C., Chung, S.: Histologic change of arteriovenous malformations of the face and scalp after free flap transfer. Plast Reconstr Surg 2000; 106: 87-93.

27. Terada, T., Higashida, R.T., Halboch, V.V., Dowd, C.F., Tsuura, M., Komai, N., Wilson, C.B., Hieshima, G.M.: Development of acquired arteriovenous fistulas in rats due to venous hypertension. J Neurosurg 80 1994; 80: 884-889.

28. Tornambe, R., Antell, D., Castellano, M.: Arteriovenous fistulae following hair transplantation: collective review and report of a case. Ann Plast Surg 1994; 33: 214-215.

29. Wilkinson, H.A.: Recurrence of vascular malformation of the scalp 18 years following excision. Case report. J Neurosurg 1971; 34: 435-437.

30. Yamanouchi, H., Hirano, S., Sakuragawa, N., Kurokawa, T.: Cerebellar arteriovenous malformation associated with facial hemangioma and arteriovenous fistula of the scalp. No To Hattatsu 1992; 24: 291-293.

García-Conde, M.; Martín-Viota, L.; Febles-García, P.; Cortés-Franco, S.; Millán-Corada, A.M.; Spreafico-Guerrero, M.; Prada, E.; García-Marín, V.: Malformación arteriovenosa gigante de cuero cabelludo: caso clínico. Neurocirugía 2006; 17: 445-449.

Correspondencia postal: Mario García Conde. Servicio de Neurocirugía. Hospital Universitario de Canarias. Ofra s/n. La Cuesta. 38320 La Laguna. Santa Cruz de Tenerife. España. 